\title{
Suggestions pour une faculté de médecine qui désire faire une réforme
}

Monsieur,

En complément de deux précédentes contributions publiées dans ces colonnes ${ }^{[1,2]}, \mathrm{j}^{\prime}$ aimerais ajouter quelques lignes de conclusion concernant la problématique de la pertinence et de l'efficacité de la formation médicale.

Considérant que les citoyens doivent être protégés par des examens de certification, vérifiant l'acquisition de compétences professionnelles, considérant que parmi les éléments qui motivent les étudiants, partout et depuis toujours, les examens de certification jouent un rôle très important et donnent le droit d'exercice, considérant que les étudiants sont supposés devenir professionnellement compétents grâce à (et trop souvent malgré) un programme de base, il est suggéré :

- de construire un «examen» (ensemble d'outils d'évaluation que l'on divise en deux parties égales, l'une destinée à usage formatif et l'autre à usage certificatif) capable de mesurer, avec un niveau adéquat de validité, la performance de toutes les compétences professionnelles (dérivées de la réalité de la profession ou adaptées de catalogues existants répondant aux besoins de la population (pertinence);

- de mettre ces outils à la libre disposition des étudiants sous forme d'évaluation formative;

- d'utiliser ces outils sous forme d'épreuves de certification pour vérifier le gain de compétence professionnelle des étudiants, entre leur entrée à l'université et la fin de leur cursus, leur donnant le droit de se présenter à un examen national éventuel et de continuer en formation continue ;

- de laisser aux enseignants la liberté individuelle d'enseigner «quoi » et «comment» selon leur choix ;

- d'identifier des lieux d'observation et de pratique (pour des stages dans la communauté, dans des structures extrahospitalières et dans des hôpitaux) où ont lieu des activités supervisées de soins (compétences professionnelles diagnostiques, thérapeutiques, de promotion de la santé, de prévention et/ou de réhabilitation, de recherche, etc.);

- de laisser la liberté aux étudiants de suivre ou non l'enseignement proposé et le libre choix de lieux d'apprentissage compte tenu de leur performance lors des évaluations formatives et jusqu'à ce qu'ils aient démontré la maîtrise des compétences professionnelles identiques à celles de l'épreuve de certification ;

- ... et pourquoi ne pas recommencer ce processus après un certain nombre d'années comme pour les pilotes des compagnies aériennes?

La mise en application de ces suggestions serait facilitée en prenant la précaution élémentaire de former une équipe cohérente d' " enseignants », chargée du choix et de la mise en œuvre de l'orientation pédagogique pour former des médecins compétents. En prime, les « compétences pédagogiques » de ces «enseignants » pourraient faire l'objet d'une attention particulière afin de contribuer à leur indispensable leadership pédagogique.

Jean-Jacques GUILBERT 5 avenue du Mail, 1205 Genève, Suisse Mailto : guilbertjj@yahoo.fr

\section{Références}

1. Guilbert J-J. Vers une formation pertinente des personnels de santé. Pourquoi cela prend-t-il du temps ? Que faire ? Pédagogie Médicale 2003;4:35-41

2. Guilbert J-J. Quelques réflexions sur les curriculums Canada Dry $^{\circledR}$. Pédagogie Médicale 2006; 7:53-8. 